\title{
Stopping a viscous fluid by a feedback dissipative external field: I. The stationary Stokes problem
}

\author{
S.N. Antontsev \\ Universidade da Beira Interior \\ Covilhã, Portugal \\ anton@ubi.pt
}

\author{
J.I. Díaz \\ Universidad Complutense \\ Madrid, Spain \\ ji_diaz@mat.ucm.es
}

\author{
H.B. de Oliveira \\ Universidade do Algarve \\ Faro, Portugal \\ holivei@ualg.pt
}

November 10, 2002

\begin{abstract}
In this work we consider a planar stationary flow of an incompressible viscous fluid in a semi-infinite strip governed by the standard Stokes system. We show how this fluid can be stopped at a finite distance of the entrance of the semi-infinite strip by means of a feedback source depending in a sub-linear way on the velocity field. This localization effect is proved reducing the problem to a non-linear bi-harmonic type one for which the localization of solutions is obtained by means of the application of an energy method, in the spirit of the monograph by Antontsev, Díaz and Shmarev [5]. Since the presence of the non-linear terms defined by the source is not standard in the fluid mechanics literature, we establish also some results about the existence and uniqueness of weak solutions for this problem.
\end{abstract}

Keywords: Stokes system, feedback dissipative field, non linear higher order equation, energy method, localization effect.

\section{Introduction}

In this paper, we present the detailed version of our note [2]. We study the planar stationary flow of an incompressible viscous fluid in a semi-infinite strip $\Omega=(0, \infty) \times(0, L), L>0$, of velocity $\mathbf{u}(\mathbf{x})=(u(\mathbf{x}), v(\mathbf{x})), \mathbf{x}=(x, y) \in \Omega$, with a non-zero velocity at the strip entrance

$$
\mathbf{u}(0, y)=\mathbf{u}_{*}(y), y \in(0, L)
$$


and zero velocity on the lateral wall

$$
\mathbf{u}(x, 0)=\mathbf{u}(x, L)=\mathbf{0}, x \in(0, \infty) .
$$

Since $\Omega$ is unbounded, we have to prescribe the velocity at infinity. We are interested in the case

$$
|\mathbf{u}(x, y)| \rightarrow 0 \text {, as } x \rightarrow \infty \text { and } y \in(0, L) .
$$

The main question we shall consider in this paper can be stated in the following terms: can we find an external localized force field $\mathbf{f}$ stopping the fluid at a finite distance, i.e., such that

$$
\mathbf{u}(x, y)=\mathbf{0} \text { for } x \geq x_{\mathbf{u}} \text { and } y \in(0, L)
$$

for some $x_{\mathbf{u}}>0$ ? In the following, we shall denote this property as the localization effect. Here, the localization of the external field must be understood in the sense that we search for a field $\mathbf{f}$ such that

$$
\mathbf{f}(\mathbf{x}, \mathbf{u})=\mathbf{0} \text { for } x \geq x_{\mathbf{f}} \text { and } y \in(0, L)
$$

for some $x_{\mathbf{f}}>0$.

Once the inertia terms are neglected, the velocity $\mathbf{u}$ and pressure $p$ are determined from the standard Stokes system

$$
\begin{gathered}
-\nu \Delta \mathbf{u}=\mathbf{f}-\nabla p \quad \text { in } \Omega, \\
\operatorname{div} \mathbf{u}=0 \quad \text { in } \Omega,
\end{gathered}
$$

where $p=p(x, y)$ stands for the hydrostatic pressure divided by the constant density of the fluid and $\nu$ is the kinematic viscosity coefficient.

We recall that due to the incompressibility condition (1.5), the first component of $\mathbf{u}_{*}(y)=\left(u_{*}(y), v_{*}(y)\right)$ must satisfy

$$
\int_{0}^{L} u_{*}(s) d s=0 .
$$

We also assume the compatibility conditions

$$
\mathbf{u}_{*}(0)=\mathbf{u}_{*}(L)=\mathbf{0} .
$$

Further on, we shall denote problem (1.1)-(1.7) as $\mathcal{P}\left(\Omega, \mathbf{u}_{*}, \mathbf{f}\right)$.

We point out the resemblance between our formulation and the important question of the confinement of a plasma, typical of magnetohydrodynamics (MHD). Although most of the studies on this effect deal with the case of the ideal MHD (i.e., with a non-viscous fluid), we recall (see, e.g., Freidberg [17]) that the 3d-MHD system involves, among other equations, the system

$$
\begin{gathered}
-\nu \triangle \mathbf{v}+\nabla p=\mathbf{j} \times \mathbf{B} \\
\mathbf{E}+\mathbf{v} \times \mathbf{B}=\eta \mathbf{j},
\end{gathered}
$$


where the resistivity $\eta$ is assumed to be a given positive constant. Now, if we assume a planar flow $(\mathbf{v}=(\mathbf{u}, 0))$ and the electric and magnetic fields $\mathbf{E}$ and $\mathbf{B}$ are given in the form $\mathbf{E}(\mathbf{x})=(0,0, E(\mathbf{x}))$ and $\mathbf{B}(\mathbf{x})=(0, B(\mathbf{x}), 0)$, then the conservation of momentum equation becomes

$$
-\nu \triangle \mathbf{u}+\nabla p=-\eta(E B, 0)-\eta\left(B^{2} u, 0\right) .
$$

So, the resultant external field is a dissipative feedback field, (i.e., it depends on its own solution $\mathbf{u}$ )

$$
\mathbf{f}=\mathbf{f}(\mathbf{x}, \mathbf{u})=-\eta(E(\mathbf{x}) B(\mathbf{x}), 0)-\eta\left(B^{2}(\mathbf{x}) u, 0\right) .
$$

Nevertheless, we shall show (see Remark 3.3) that this field (when $E(\mathbf{x})$ and $B(\mathbf{x})$ are assumed known) is not able to lead to a positive answer to our question: the $x$-decay of $\mathbf{u}$ is of exponential type. The results on confinement in toroidal devices (as Tokamaks and Stellarators) assume that $\mathbf{u} \equiv \mathbf{0}$ and are based on the so called Grad-Safranov equation for the potential function of the magnetic field (see, e.g., Berstycki and Brezis [9], Temam [27] and Simon [26] for Tokamaks and Díaz and Rakotoson [13] for Stellarators).

We also recall that for the classical Stokes problem, i.e., with $\mathbf{f}=\mathbf{f}(\mathbf{x})$ prescribed, it is well known that the decay is exponential and so the localization effect fails. See Knowles [22], for the exponential decay results on the Saint-Venant's Principle in the two-dimensional linear theory of elasticity, which can be easily adapted for the problem $\mathcal{P}\left(\Omega, \mathbf{u}_{*}, \mathbf{f}\right)$. See also Toupin [28] and Galdi [18], Chapter VI.

The main goal of this paper is to show the localization effect when we assume the external body forces be given in feedback form, $\mathbf{f}: \Omega \times \mathbb{R}^{2} \rightarrow \mathbb{R}^{2}, \mathbf{f}(\mathbf{x}, \mathbf{u})=$ $\left(f_{1}(\mathbf{x}, \mathbf{u}), f_{2}(\mathbf{x}, \mathbf{u})\right)$, and are such that, for every $\mathbf{u} \in \mathbb{R}^{2}, \mathbf{u}=(u, v)$, and for almost all $\mathrm{x} \in \Omega$,

$$
-\mathbf{f}(\mathbf{x}, \mathbf{u}) \cdot \mathbf{u} \geq \delta \chi_{\mathbf{f}}(\mathbf{x})|u|^{1+\sigma}-g(\mathbf{x})
$$

for some $\delta>0,0<\sigma<1$ and

$$
g \in \mathrm{L}^{1}\left(\Omega^{x_{g}}\right), g \geq 0, g(\mathbf{x})=0 \text { a.e. in } \Omega_{x_{g}}
$$

for some $x_{\mathbf{f}}, x_{g}$ with $0 \leq x_{g} \leq x_{\mathbf{f}} \leq \infty$ and $x_{\mathbf{f}}$ large enough, where $\Omega^{x_{g}}=$ $\left(0, x_{g}\right) \times(0, L)$ and $\Omega_{x_{g}}=\left(x_{g}, \infty\right) \times(0, L)$. The function $\chi_{\mathbf{f}}$ denotes the characteristic function of the interval $\left(0, x_{\mathbf{f}}\right)$, i.e., $\chi_{\mathbf{f}}(\mathbf{x})=1$, if $x \in\left(0, x_{\mathbf{f}}\right)$ and $\chi_{\mathbf{f}}(\mathbf{x})=0$, if $x \notin\left(0, x_{\mathbf{f}}\right)$. Notice that such a result will hold, in particular, for the simpler non-localized case corresponding to $x_{\mathbf{f}}=\infty$ (and so $\left.\chi_{\mathbf{f}}(\mathbf{x}) \equiv 1\right)$. As already mentioned, the answer becomes negative for $\sigma=1$. For some comments on the other limit case, $\sigma=0$, see Remark 3.4.

We recall that other localization effects as, for instance, the finite extinction time or the finite speed of propagations for some problems on viscous fluids of different natures, as well for some problems on non-viscous fluids have been established (see [5] and the references therein). In [5] the finite extinction time property was established for pseudo-plastic fluids, i.e., a class of non-Newtonian 
fluids satisfying a certain non-linearity condition on the stress tensor. To the best of our knowledge, the only result on the finite speed of propagations property, for the solution of a fluid mechanics formulation is due to [5], Section 4.7.5. In that case, for a dilatant fluid in a pipe, a certain non-linearity condition on the source $\mathbf{f}$ was also very important there.

The localization effect will be proved for weak solutions of the problem $\mathcal{P}\left(\Omega, \mathbf{u}_{*}, \mathbf{f}\right)$ under assumptions (1.8) and (1.9). Since the presence of the nonlinear terms defined by $\mathbf{f}(\mathbf{x}, \mathbf{u})$ is not standard in the fluid mechanics literature, we collect in Section 2 some results about the existence and uniqueness of weak solutions for problem $\mathcal{P}\left(\Omega, \mathbf{u}_{*}, \mathbf{f}\right)$.

The localization effect is proved, in Section 3, by means of the application of an energy method to the associated current function $\psi\left(\mathbf{u}=\left(\psi_{y},-\psi_{x}\right)\right)$. The function $\psi$ satisfies the following higher order non-linear equation and boundary conditions

$$
\left\{\begin{array}{l}
\nu \triangle^{2} \psi+\frac{\partial f_{1}}{\partial y}\left(\mathbf{x}, \psi_{y},-\psi_{x}\right)-\frac{\partial f_{2}}{\partial x}\left(\mathbf{x}, \psi_{y},-\psi_{x}\right)=0 \text { in } \Omega, \\
\psi(x, 0)=\psi(x, L)=0 \text { for } x \in(0, \infty) \\
\frac{\partial \psi}{\partial n}(x, 0)=\frac{\partial \psi}{\partial n}(x, L)=0 \text { for } x \in(0, \infty), \\
\psi(0, y)=\int_{0}^{y} u_{*}(s) d s, \frac{\partial \psi}{\partial n}(0, y)=v_{*}(y) \text { for } y \in(0, L), \\
\psi(x, y),|\nabla \psi(x, y)| \rightarrow 0 \text { as } x \rightarrow \infty \text { and for } y \in(0, L) .
\end{array}\right.
$$

Further on, we shall denote problem (1.10) as $\mathcal{P}_{\psi}$.

We shall adapt in the paper the half-planes technique introduced in Bernis [6] for the study of other higher order equations. We point out that in contrast with the problems considered in the mentioned work, (1.10) do not contains any zero order term. Our approach is inspired by some previous unidirectional results for anisotropic equations proved in [5], Section 1.4.2, by using a different energy method.

In a final section, we show that the localization effect holds also for the case of non-constant semi-infinite strip domains of the type $\Omega=(0, \infty) \times\left(L_{1}(x), L_{2}(x)\right)$.

The consideration of the stationary and transient problem for the NavierStokes system will be developed by the authors in the future papers ( [3] and $[4])$.

\section{On the existence and uniqueness of weak so- lutions.}

In this section we consider a general class of domains of the form $\Omega=(0, \infty) \times$ $\left(L_{1}(x), L_{2}(x)\right)$ with

$$
\begin{gathered}
L_{1}, L_{2} \in \mathrm{C}^{2}(0, \infty), \\
\frac{1}{L} \leq\left|L_{2}(x)-L_{1}(x)\right| \leq L,
\end{gathered}
$$

for all $x \geq 0$, where $L$ is a positive constant. Now, the boundary conditions (1.1) and (1.2) are replaced, respectively, by

$$
\mathbf{u}(0, y)=\mathbf{u}_{*}(y), y \in\left(L_{1}(0), L_{2}(0)\right),
$$




$$
\mathbf{u}\left(x, L_{1}(x)\right)=\mathbf{u}\left(x, L_{2}(x)\right)=\mathbf{0}, x \in(0, \infty),
$$

where $\mathbf{u}_{*}(y)=\left(u_{*}(y), v_{*}(y)\right)$ satisfies the compatibility conditions

$$
\int_{L_{1}(0)}^{L_{2}(0)} u_{*}(s) d s=0
$$

and

$$
\mathbf{u}_{*}\left(L_{1}(0)\right)=\mathbf{u}_{*}\left(L_{2}(0)\right)=\mathbf{0} .
$$

We shall search solutions such that $\int_{\Omega}|\nabla \mathbf{u}|^{2} d \mathbf{x}<\infty$. Moreover, due to the fact that the Poincaré inequality

$$
\int_{L_{1}(x)}^{L_{2}(x)}|u|^{p} d y \leq\left(\frac{L}{\pi}\right)^{p} \int_{L_{1}(x)}^{L_{2}(x)}\left|u^{\prime}\right|^{p} d y
$$

holds, where $L=\sup _{x \in[0, \infty)}\left|L_{2}(x)-L_{1}(x)\right|$, for every $u \in \mathrm{W}_{0}^{1, p}\left(L_{1}(x), L_{2}(x)\right)$ and $1 \leq p<\infty$ (see, e.g., Gilbarg and Trudinger [19], Section 7.8), our searched solution will be an element of the Sobolev space $\mathbf{H}^{1}(\Omega)$ simplifying, in this way, the functional framework needed for other unbounded domains.

In order to define the notion of a weak solution, we introduce the functional spaces

$$
\begin{gathered}
\widetilde{\mathbf{H}}(\Omega)=\left\{\mathbf{u} \in \mathbf{H}^{1}(\Omega): \operatorname{div} \mathbf{u}=0, \mathbf{u}(0, .)=\mathbf{u}_{*}(.),\right. \\
\left.\mathbf{u}\left(x, L_{1}(x)\right)=\mathbf{u}\left(x, L_{2}(x)\right)=\mathbf{0}, x \in(0, \infty), \lim _{x \rightarrow \infty}|\mathbf{u}|=0\right\}
\end{gathered}
$$

and

$$
\begin{gathered}
\widetilde{\mathbf{H}}_{0}(\Omega)=\left\{\mathbf{u} \in \mathbf{H}^{1}(\Omega): \operatorname{div} \mathbf{u}=0, \mathbf{u}(0, .)=\mathbf{0},\right. \\
\left.\mathbf{u}\left(x, L_{1}(x)\right)=\mathbf{u}\left(x, L_{2}(x)\right)=\mathbf{0}, x \in(0, \infty), \lim _{x \rightarrow \infty}|\mathbf{u}|=0\right\}
\end{gathered}
$$

We shall assume that

$$
\mathbf{u}_{*} \in \mathbf{H}^{\frac{1}{2}}\left(L_{1}(0), L_{2}(0)\right),
$$

where we used the notation $\mathbf{H}^{\frac{1}{2}}=\mathbf{W}^{\frac{1}{2}, 2}$. For more details on this space see, e.g., [18], Chapter II.

In this section we shall assume that $\mathbf{f}: \Omega \times \mathbb{R}^{2} \rightarrow \mathbb{R}^{2}$, with $\mathbf{f}(\mathbf{x}, \mathbf{u})=$ $\left(f_{1}(\mathbf{x}, \mathbf{u}), f_{2}(\mathbf{x}, \mathbf{u})\right), \mathbf{u}=(u, v)$,

$$
\mathbf{f}(\mathbf{x}, \mathbf{u})=-\delta \chi_{\mathbf{f}}(\mathbf{x})\left(|u|^{\sigma-1} u, 0\right)-\mathbf{h}(\mathbf{x}, \mathbf{u}),
$$

for some $\delta>0,0 \leq x_{\mathbf{f}} \leq \infty$ and $0<\sigma<1$. Here, $\mathbf{h}(\mathbf{x}, \mathbf{u})$ is a Carathéodory function, i.e., $\mathbf{h}(\mathbf{x}, \mathbf{u})$ is measurable in $\mathbf{x}$ for all $\mathbf{u} \in \mathbb{R}^{2}$ and continuous in $\mathbf{u}$ for almost all $\mathbf{x} \in \Omega$, such that

$$
\mathbf{h}(\mathbf{x}, \mathbf{u}) \cdot \mathbf{u} \geq-g(\mathbf{x}), \text { for every } \mathbf{u} \in \mathbb{R}^{2} \text { and a.a. } \mathbf{x} \in \Omega,
$$

for some

$$
g \in \mathrm{L}^{1}\left(\Omega^{x_{g}}\right), g \geq 0, g(\mathbf{x})=0 \text { a.e. in } \Omega_{x_{g}},
$$


with $0 \leq x_{g}<x_{\mathbf{f}}$, and

$$
H_{M} \in \mathrm{L}^{1}\left(\Omega^{x_{\mathbf{f}}}\right), \text { for all } M>0,
$$

where $H_{M}(\mathbf{x})=\sup _{|\mathbf{u}| \leq M}|\mathbf{h}(\mathbf{x}, \mathbf{u})|$.

Notice that no upper restriction on the growth of $|\mathbf{f}(\mathbf{x}, \mathbf{u})|$ with respect to $\mathbf{u}$ is imposed. This is the reason why sometimes this type of terms is called as a strong nonlinearity.

Definition 2.1 We say that a vector function $\mathbf{u}$ is a weak solution of problem $\mathcal{P}\left(\Omega, \mathbf{u}_{*}, \mathbf{f}\right)$, if:

(i) $\mathbf{u} \in \widetilde{\mathbf{H}}(\Omega), \mathbf{f}(\mathbf{x}, \mathbf{u}) \in \mathbf{L}^{1}(\Omega)$;

(ii) $\nu \int_{\Omega} \nabla \mathbf{u}: \nabla \boldsymbol{\varphi} d \mathbf{x}=\int_{\Omega} \mathbf{f} \cdot \boldsymbol{\varphi} d \mathbf{x}$, for all $\boldsymbol{\varphi} \in \widetilde{\mathbf{H}}_{0}(\Omega) \cap \mathbf{L}^{\infty}(\Omega)$ with compact support.

Later on, in our considerations, there will appear two types of constants: absolute constants, which will be denoted by the letter $C$ and constants depending on parameters, which will be numbered by subscripts and will contain the parameters in parenthesis.

The main result of this section is as follows.

Theorem 2.1 Under the above assumptions on $\mathbf{f}(\mathbf{x}, \mathbf{u})$, there exists, at least, one weak solution of problem $\mathcal{P}\left(\Omega, \mathbf{u}_{*}, \mathbf{f}\right)$. Moreover, $\mathbf{f}(\mathbf{x}, \mathbf{u}) \cdot \mathbf{u}$ lies in $\mathrm{L}^{1}(\Omega)$ and $\mathbf{u}$ satisfies to the energy estimate

$$
\begin{gathered}
\int_{\Omega}\left(|\nabla \mathbf{u}|^{2}+\chi_{\mathbf{f}}|u|^{1+\sigma}+|\mathbf{h}(\mathbf{x}, \mathbf{u}) \cdot \mathbf{u}|\right) d \mathbf{x} \leq \\
C_{1}(L, \delta, \nu, \sigma)\left(\left\|\mathbf{u}_{*}\right\|_{\mathbf{H}^{\frac{1}{2}}\left(L_{1}(0), L_{2}(0)\right)}^{2}+\|g\|_{L^{1}\left(\Omega^{x^{g}}\right)}+1\right) .
\end{gathered}
$$

Problem $\mathcal{P}\left(\Omega, \mathbf{u}_{*}, \mathbf{f}\right)$ has only one solution, if, in addition, the inequality

$$
\left(\mathbf{f}\left(\mathbf{x}, \mathbf{u}_{1}\right)-\mathbf{f}\left(\mathbf{x}, \mathbf{u}_{2}\right)\right) \cdot\left(\mathbf{u}_{1}-\mathbf{u}_{2}\right) \leq 0
$$

holds for every $\mathbf{u}_{1}, \mathbf{u}_{2} \in \mathbb{R}^{2}$ and almost all $\mathbf{x} \in \Omega$.

Proof. We split the proof into two parts: existence and uniqueness.

Existence. First step. We start by considering the auxiliary problem, in $\Omega^{N}=(0, N) \times\left(L_{1}(x), L_{2}(x)\right)$, with $N \in \mathbb{N}$ given,

$$
\begin{cases}-\nu \Delta \mathbf{u}^{N}=\mathbf{f}-\boldsymbol{\nabla} p^{N} & \text { in } \Omega^{N} \\ \operatorname{div} \mathbf{u}^{N}=0 & \text { for } x \in(0, N) \\ \mathbf{u}^{N}\left(x, L_{1}(x)\right)=\mathbf{u}^{N}\left(x, L_{2}(x)\right)=\mathbf{0} & \text { for } y \in\left(L_{1}(0), L_{2}(0)\right), \\ \mathbf{u}^{N}(0, y)=\mathbf{u}_{*}(y) & \text { for } y \in\left(L_{1}(N), L_{2}(N)\right) \\ \mathbf{u}^{N}(N, y)=\mathbf{0} & \end{cases}
$$

which will be denoted by $\mathcal{P}\left(\Omega^{N}, \mathbf{u}_{*}, \mathbf{f}\right)$, wether $\mathbf{f} \equiv \mathbf{f}(\mathbf{x})$ or $\mathbf{f} \equiv \mathbf{f}\left(\mathbf{x}, \mathbf{u}^{N}\right)$. With no loss of generality, we assume $N>1$, and let $\mathbf{U}^{1}$ be an extension of $\mathbf{u}_{*}$ to 
$\Omega^{1}=(0,1) \times\left(L_{1}(x), L_{2}(x)\right)$ such that: (i) $\mathbf{U}^{1} \in \mathbf{H}^{1}\left(\Omega^{1}\right)$; (ii) $\operatorname{div} \mathbf{U}^{1}=0$ in $\Omega^{1}$; (iii) $\mathbf{U}^{1}=\mathbf{u}_{*}$ on $x=0, \mathbf{U}^{1}=\mathbf{0}$ on $x=1$ and on $y=L_{1}(x), y=L_{2}(x)$, for $x \in(0,1)$, in the trace sense. Such an extension exists (see , e.g., [18], Section III.3), and, moreover, $\left\|\mathbf{U}^{1}\right\|_{\mathbf{H}^{1}\left(\Omega^{1}\right)} \leq C_{2}(L)\left\|\mathbf{u}_{*}\right\|_{\mathbf{H}^{\frac{1}{2}}\left(L_{1}(0), L_{2}(0)\right)}$.

Now, we consider the extension $\mathbf{U}^{N}$ to $\Omega^{N}$ such that $\mathbf{U}^{N}=\mathbf{U}^{1}$ if $x<1$ and $\mathbf{U}^{N}=\mathbf{0}$ if $x \geq 1$. From what we had said above, $\mathrm{U}^{N} \in \mathbf{H}^{1}\left(\Omega^{N}\right)$ and we have

$$
\left\|\mathbf{U}^{N}\right\|_{\mathbf{H}^{1}\left(\Omega^{N}\right)} \leq C_{3}(L)\left\|\mathbf{u}_{*}\right\|_{\mathbf{H}^{\frac{1}{2}}\left(L_{1}(0), L_{2}(0)\right)} .
$$

Moreover, using Hölder's inequality, one can prove

$$
\int_{\Omega^{N}}\left|\mathbf{U}^{N}\right|^{p} d \mathbf{x} \leq C_{4}(L, p)\left\|\mathbf{u}_{*}\right\|_{\mathbf{H}^{\frac{1}{2}}\left(L_{1}(0), L_{2}(0)\right)}^{p}, \quad \text { for } 1 \leq p<2 .
$$

Second step. Firstly, we consider the intermediary case in which we assume, additionally,

$$
|\mathbf{h}(\mathbf{x}, \mathbf{u})| \leq C \chi_{\mathbf{f}}(\mathbf{x})
$$

for some positive constant $C$, for all $\mathbf{u} \in \mathbb{R}^{2}$ and almost all $\mathbf{x} \in \Omega$.

We recall that, if we consider the linear problem $\mathcal{P}\left(\Omega^{N}, \mathbf{u}_{*}, \mathbf{f}\right)$ with $\mathbf{f} \equiv \mathbf{f}(\mathbf{x})$ given arbitrarily, for instance, $\mathbf{f} \in \mathbf{L}^{2}\left(\Omega^{N}\right)$, then we know the existence of a unique weak solution $\mathbf{u}^{N} \in \mathbf{H}^{1}\left(\Omega^{N}\right)$ (see, e.g., [18], Chapter IV) which satisfies to the energy relation

$$
\nu \int_{\Omega^{N}} \nabla \mathbf{u}^{N}:\left(\nabla \mathbf{u}^{N}-\nabla \mathbf{U}^{N}\right) d \mathbf{x}=\int_{\Omega^{N}} \mathbf{f} \cdot\left(\mathbf{u}^{N}-\mathbf{U}^{N}\right) d \mathbf{x} .
$$

Applying modules to the right-hand side, next using Young's and Poincaré's (2.17) inequalities and then (2.24), we obtain the following estimate

$$
\left\|\mathbf{u}^{N}\right\|_{\mathbf{H}^{1}\left(\Omega^{N}\right)}^{2} \leq C_{5}(L, \nu)\left(\left\|\mathbf{u}_{*}\right\|_{\mathbf{H}^{\frac{1}{2}\left(L_{1}(0), L_{2}(0)\right)}}^{2}+\|\mathbf{f}\|_{\mathbf{L}^{2}\left(\Omega^{N}\right)}^{2}\right) .
$$

We look for a weak solution $\mathbf{u}^{N}$ of the non-linear problem $\mathcal{P}\left(\Omega^{N}, \mathbf{u}_{*}, \mathbf{f}\right)$, with $\mathbf{f} \equiv \mathbf{f}\left(\mathbf{x}, \mathbf{u}^{N}\right)$. Notice that, in the special case of $(2.26), \mathbf{f}\left(\mathbf{x}, \mathbf{u}^{N}\right)$ satisfies

$$
\left|\mathbf{f}\left(\mathbf{x}, \mathbf{u}^{N}\right)\right| \leq a(\mathbf{x})\left|\mathbf{u}^{N}\right|^{\sigma}+b(\mathbf{x})
$$

for every $\mathbf{u}^{N} \in \mathbb{R}^{2}$ and almost all $\mathbf{x} \in \Omega^{N}$, where $a, b \geq 0$ are measurable functions in $\Omega^{N}$ with

$$
a \in \mathrm{L}^{\frac{2}{1-\sigma}}\left(\Omega^{N}\right), b \in \mathrm{L}^{2}\left(\Omega^{N}\right) .
$$

From (2.29) and after made use of Young's inequality with a suitable $\varepsilon$, we have

$$
\left|\mathbf{f}\left(\mathbf{x}, \mathbf{u}^{N}\right)\right|^{2} \leq 4\left[\varepsilon\left|\mathbf{u}^{N}\right|^{2}+(1-\sigma)\left(\frac{\sigma}{\varepsilon}\right)^{\frac{\sigma}{1-\sigma}} a^{\frac{2}{1-\sigma}}+b^{2}\right] .
$$


Gathering (2.28), (2.31), using (2.24) and choosing $\varepsilon$ in a reasonable way, we obtain the a priori estimate for the non-linear problem $\mathcal{P}\left(\Omega^{N}, \mathbf{u}_{*}, \mathbf{f}\left(\mathbf{x}, \mathbf{u}^{N}\right)\right)$

$$
\left\|\mathbf{u}^{N}\right\|_{\mathbf{H}^{1}\left(\Omega^{N}\right)}^{2} \leq C_{6}\left(\|a\|_{\mathrm{L}^{1-\sigma}\left(\Omega^{N}\right)}^{\frac{2}{1-\sigma}}+\|b\|_{L^{2}\left(\Omega^{N}\right)}^{2}+\left\|\mathbf{u}_{*}\right\|_{\mathbf{H}^{\frac{1}{2}}\left(L_{1}(0), L_{2}(0)\right)}^{2}\right),
$$

with $C_{6}=C_{6}(L, \nu, \sigma)$. Next, given $\varepsilon>0$ and $\mathbf{v}^{N} \in \mathbf{L}^{2}\left(\Omega^{N}\right)$, we define

$$
\mathbf{v}_{\varepsilon}^{N}=\frac{\mathbf{v}^{N}}{1+\varepsilon\left\|\mathbf{v}^{N}\right\|_{\mathbf{L}^{2}\left(\Omega^{N}\right)}} .
$$

Obviously,

$$
\left\|\mathbf{v}_{\varepsilon}^{N}\right\|_{\mathbf{L}^{2}\left(\Omega^{N}\right)} \leq \min \left(\frac{1}{\varepsilon},\left\|\mathbf{v}^{N}\right\|_{\mathbf{L}^{2}\left(\Omega^{N}\right)}\right)
$$

and consequently $\mathbf{v}_{\varepsilon}^{N} \in \mathbf{L}^{2}\left(\Omega^{N}\right)$. We consider the linear problem $\mathcal{P}\left(\Omega^{N}, \mathbf{u}_{*}, \mathbf{f}\right)$ with $\mathbf{f}(\mathbf{x})=\mathbf{f}\left(\mathbf{x}, \mathbf{v}_{\varepsilon}^{N}\right)$. The above considerations yield that for each $\mathbf{v}_{\varepsilon}^{N} \in$ $\mathbf{L}^{2}\left(\Omega^{N}\right)$, there exists a unique weak solution $\mathbf{u}_{\varepsilon}^{N} \in \mathbf{H}^{1}\left(\Omega^{N}\right)$. Thus, we can define a non-linear operator $\Lambda: \mathbf{L}^{2}\left(\Omega^{N}\right) \rightarrow \mathbf{L}^{2}\left(\Omega^{N}\right)$ by setting

$$
\Lambda\left(\mathbf{v}_{\varepsilon}^{N}\right)=\mathbf{u}_{\varepsilon}^{N} .
$$

According to (2.32),

$$
\left\|\boldsymbol{\Lambda}\left(\mathbf{v}_{\varepsilon}^{N}\right)\right\|_{\mathbf{L}^{2}\left(\Omega^{N}\right)}^{2}=\left\|\mathbf{u}_{\varepsilon}^{N}\right\|_{\mathbf{L}^{2}\left(\Omega^{N}\right)}^{2} \leq\left\|\mathbf{u}_{\varepsilon}^{N}\right\|_{\mathbf{H}^{1}\left(\Omega^{N}\right)}^{2}<R_{\varepsilon}
$$

where

$$
R_{\varepsilon}=C_{6}(L, \nu, \sigma)\left(\|a\|_{\mathrm{L}^{\frac{2}{1-\sigma}}\left(\Omega^{N}\right)}^{\frac{2}{1-\sigma}}+\|b\|_{\mathrm{L}^{2}\left(\Omega^{N}\right)}^{2}+\left\|\mathbf{u}_{*}\right\|_{\mathbf{H}^{\frac{1}{2}}\left(L_{1}(0), L_{2}(0)\right)}^{2}+\frac{1}{\varepsilon}\right) .
$$

Under the assumptions (2.18) and (2.30) and from (2.34), the operator (2.33) maps $\mathbf{L}^{2}\left(\Omega^{N}\right)$ into a bounded subset of $\mathbf{H}^{1}\left(\Omega^{N}\right)$ and from the Sobolev compact embedding $\mathbf{H}^{1}\left(\Omega^{N}\right) \rightarrow \mathbf{L}^{2}\left(\Omega^{N}\right)$, it is a completely continuous operator. According to Shauder's theorem, the operator (2.33) has a fixed point

$$
\mathbf{u}_{\varepsilon}^{N}=\boldsymbol{\Lambda}\left(\mathbf{u}_{\varepsilon}^{N}\right)
$$

for any $\varepsilon>0$. On the other hand, using (2.32), we obtain the estimate

$$
\left\|\mathbf{u}_{\varepsilon}^{N}\right\|_{\mathbf{H}^{1}\left(\Omega^{N}\right)}^{2} \leq C_{7}\left(\|a\|_{\mathrm{L}^{1-\sigma}\left(\Omega^{N}\right)}^{\frac{2}{1-\sigma}}+\|b\|_{\mathrm{L}^{2}\left(\Omega^{N}\right)}^{2}+\left\|\mathbf{u}_{*}\right\|_{\mathbf{H}^{\frac{1}{2}}\left(L_{1}(0), L_{2}(0)\right)}^{2}\right),
$$

with $C_{7}=C_{7}(L, \nu, \sigma)$. Let us now consider a sequence $\mathbf{u}_{\varepsilon, k}^{N} \in \mathbf{H}^{1}\left(\Omega^{N}\right)$, with $k \in \mathbb{N}$, satisfying (2.27) with $\mathbf{f}(\mathbf{x})$ replaced by $\mathbf{f}\left(\mathbf{x}, \mathbf{u}_{\varepsilon, k}^{N}\right)$. Then, from (2.35), there exists a subsequence, which we still denote by $\mathbf{u}_{\varepsilon, k}^{N}$, such that

$$
\mathbf{u}_{\varepsilon, k}^{N} \rightarrow \mathbf{u}_{\varepsilon}^{N} \text {, weakly in } \mathbf{H}^{1}\left(\Omega^{N}\right), \text { as } k \rightarrow \infty
$$


and because $\mathbf{f}$ is a Carathéodory function,

$$
\mathbf{f}\left(\mathbf{x}, \mathbf{u}_{\varepsilon, k}^{N}\right) \rightarrow \mathbf{f}\left(\mathbf{x}, \mathbf{u}_{\varepsilon}^{N}\right), \text { in } \mathbf{L}^{1}\left(\Omega^{N}\right), \text { as } k \rightarrow \infty .
$$

Then, passing to the limit in $\varepsilon \rightarrow 0$, we prove the existence of, at least, one weak solution $\mathbf{u}^{N} \in \mathbf{H}^{1}\left(\Omega^{N}\right)$ to the non-linear problem $\mathcal{P}\left(\Omega^{N}, \mathbf{u}_{*}, \mathbf{f}\left(\mathbf{x}, \mathbf{u}^{N}\right)\right)$.

Third step. We shall prove the a priori estimate (independent of $N$ ) for $\mathbf{u}^{N}$

$$
\begin{aligned}
& \int_{\Omega^{N}}\left(\left|\nabla \mathbf{u}^{N}\right|^{2}+\chi_{\mathbf{f}}\left|u^{N}\right|^{1+\sigma}+\left|\mathbf{h}\left(\mathbf{x}, \mathbf{u}^{N}\right) \cdot \mathbf{u}^{N}\right|\right) d \mathbf{x} \\
\leq & C_{8}(L, \delta, \nu, \sigma)\left(\left\|\mathbf{u}_{*}\right\|_{\mathbf{H}^{\frac{1}{2}}\left(L_{1}(0), L_{2}(0)\right)}^{2}+\|g\|_{L^{1}\left(\Omega^{x} g\right)}+1\right) .
\end{aligned}
$$

We point out that from assumptions (2.20) and (2.21),

$$
|\mathbf{h}(\mathbf{x}, \mathbf{u}) \cdot \mathbf{u}| \leq \mathbf{h}(\mathbf{x}, \mathbf{u}) \cdot \mathbf{u}+2 g(\mathbf{x})
$$

for every $\mathbf{u} \in \mathbb{R}^{2}$ and almost all $\mathbf{x} \in \Omega$.

In the energy relation (2.27) satisfied by $\mathbf{u}^{N}$, we use assumption (2.19), next we add $\left|\mathbf{h}\left(\mathbf{x}, \mathbf{u}^{N}\right) \cdot \mathbf{u}^{N}\right|$ to both sides of the resultant equation, we use assumptions (2.21) and (2.26) and, also, (2.37) and we apply Young's inequality with a suitable $\varepsilon>0$, and obtain

$$
\begin{gathered}
\int_{\Omega^{N}}\left(\left|\nabla \mathbf{u}^{N}\right|^{2}+\chi_{\mathbf{f}}\left|u^{N}\right|^{1+\sigma}+\left|\mathbf{h}\left(\mathbf{x}, \mathbf{u}^{N}\right) \cdot \mathbf{u}^{N}\right|\right) d \mathbf{x} \leq \\
C_{9}\left[\int_{\Omega^{N}} \chi_{\mathbf{f}}\left(\left|\mathbf{U}^{N}\right|+\left|\mathbf{U}^{N}\right|^{1+\sigma}\right) d \mathbf{x}+\int_{\Omega^{N}}\left|\nabla \mathbf{U}^{N}\right|^{2} d \mathbf{x}+\|g\|_{\mathrm{L}^{1}\left(\Omega^{x}\right)}\right],
\end{gathered}
$$

with $C_{9}=C_{9}(\delta, \nu, \sigma)$. Then, we use (2.24) and (2.25), and get

$$
\begin{gathered}
\int_{\Omega^{N}}\left(\left|\nabla \mathbf{u}^{N}\right|^{2}+\chi_{\mathbf{f}}\left|u^{N}\right|^{1+\sigma}+\left|\mathbf{h}\left(\mathbf{x}, \mathbf{u}^{N}\right) \cdot \mathbf{u}^{N}\right|\right) d \mathbf{x} \leq C_{10}\left(\left\|\mathbf{u}_{*}\right\|_{\mathbf{H}^{\frac{1}{2}}\left(L_{1}(0), L_{2}(0)\right)}\right. \\
\left.+\left\|\mathbf{u}_{*}\right\|_{\mathbf{H}^{\frac{1}{2}}\left(L_{1}(0), L_{2}(0)\right)}^{1+\sigma}+\left\|\mathbf{u}_{*}\right\|_{\mathbf{H}^{\frac{1}{2}}\left(L_{1}(0), L_{2}(0)\right)}^{2}+\|g\|_{\mathrm{L}^{1}\left(\Omega^{x^{x}}\right)}\right)
\end{gathered}
$$

with $C_{10}=C_{10}(L, \delta, \nu, \sigma)$. Finally, once that $0<\sigma<1$, we can use the algebraic inequality asserting that $A+A^{1+\sigma}+A^{2} \leq C\left(A^{2}+1\right)$, for every $A \geq 0$ and $C$ a positive constant, to obtain (2.36).

Fourth Step. Now, for each $N \in \mathbb{N}$, we consider a sequence $\mathbf{u}_{k}^{N}$ of weak solutions to problems $\mathcal{P}\left(\Omega^{N}, \mathbf{u}_{*}, \mathbf{f}\left(\mathbf{x}, \mathbf{u}_{k}^{N}\right)\right)$ and thus satisfying (2.36). In consequence, using a standard diagonal process and that $\mathbf{f}\left(\mathbf{x}, \mathbf{u}_{k}^{N}\right)$ is a Carathéodory function, we can choose a subsequence $\mathbf{u}_{k}^{N_{k}}$, such that

$$
\mathbf{u}_{k}^{N_{k}} \rightarrow \mathbf{u}, \quad \text { weakly in } \mathbf{H}^{1}\left(\Omega^{R}\right), \quad \text { as } k \rightarrow \infty,
$$

and

$$
\mathbf{f}\left(\mathbf{x}, \mathbf{u}_{k}^{N_{k}}\right) \rightarrow \mathbf{f}(\mathbf{x}, \mathbf{u}), \text { in } \mathbf{L}^{1}\left(\Omega^{R}\right), \text { as } k \rightarrow \infty
$$


for every $R>0$ and $\mathbf{u}$ is a weak solution to the non-linear problem $\mathcal{P}\left(\Omega, \mathbf{u}_{*}\right.$, $\mathbf{f}(\mathbf{x}, \mathbf{u})$ ), once we assume condition (2.26). In addition, $\mathbf{u}$ satisfies to the energy estimate (2.23).

Fifth step. We proceed with a truncation and approximation argument to deal with the general case, i.e., the case without condition (2.26). We shall adapt the arguments of Brezis and Browder [10] (see, also, Bernis [8]). We define the n-radial truncation $\mathbf{h}_{n}(\mathbf{x}, \mathbf{u})$ defined by

$$
\mathbf{h}_{n}(\mathbf{x}, \mathbf{u})= \begin{cases}\mathbf{h}(\mathbf{x}, \mathbf{u}) & \text { if }|\mathbf{u}| \leq n, \\ \mathbf{h}(\mathbf{x},(n \cos \theta, n \sin \theta)) & \text { if } \mathbf{u}=(r \cos \theta, r \sin \theta) \text { and } r>n .\end{cases}
$$

It was shown in Vrabie [29], Lemma 3.4.3, that $\mathbf{h}_{n}(\mathbf{x}, \mathbf{u})$ is continuous and bounded on $\mathbf{u}$ for almost all $\mathbf{x} \in \Omega$. Then

$$
\mathbf{h}_{n}\left(\mathbf{x}, \mathbf{u}_{n}\right) \rightarrow \mathbf{h}(\mathbf{x}, \mathbf{u}) \text {, for a.a. } \mathbf{x} \in \Omega \text {, as } n \rightarrow \infty .
$$

Moreover, $\mathbf{h}_{n}(\mathbf{x}, \mathbf{u})$ also satisfies assumptions (2.20)-(2.22) and (2.26) with the same functions $g$ and $H_{M}$. From Step 4, it follows that there exists a weak solution $\mathbf{u}_{n}$ of problem $\mathcal{P}\left(\Omega, \mathbf{u}_{*}, \mathbf{f}\left(\mathbf{x}, \mathbf{u}_{\mathbf{n}}\right)\right)$ and which satisfies (2.23) with $C_{1}$ independent of $n$. Therefore, there exists a subsequence, which we still denote by $\mathbf{u}_{n}$, such that

$$
\mathbf{u}_{n} \rightarrow \mathbf{u} \text {, weakly in } \mathbf{H}^{1}(\Omega) \text {, as } n \rightarrow \infty .
$$

On the other side, we also have

$$
\int_{\Omega}\left|\mathbf{h}_{n}\left(\mathbf{x}, \mathbf{u}_{n}\right) \cdot \mathbf{u}_{n}\right| d \mathbf{x} \leq C_{11},
$$

with $C_{11}$ independent of $n$. Therefore, from (2.38), (2.39) and Fatou's Lemma, $\mathbf{h}(\mathbf{x}, \mathbf{u}) \cdot \mathbf{u} \in \mathrm{L}^{1}(\Omega)$. Moreover, for any $M>0$,

$$
\left|\mathbf{h}_{n}\left(\mathbf{x}, \mathbf{u}_{n}\right)\right| \leq\left|\mathbf{h}\left(\mathbf{x}, \mathbf{u}_{n}\right)\right| \leq H_{M}(\mathbf{x}) \text {, if }\left|\mathbf{u}_{n}\right|<M .
$$

On the other hand, if $\left|\mathbf{u}_{n}\right| \geq M$, then

$$
M \int_{\Omega}\left|\mathbf{h}_{n}\left(\mathbf{x}, \mathbf{u}_{n}\right)\right|\left|\cos \beta_{n}\right| d \mathbf{x} \leq \int_{\Omega}\left|\mathbf{h}_{n}\left(\mathbf{x}, \mathbf{u}_{n}\right) \cdot \mathbf{u}_{n}\right| d \mathbf{x} \leq C_{11},
$$

where $\beta_{n}(\mathbf{x})=\operatorname{angle}\left(\mathbf{h}_{n}\left(\mathbf{x}, \mathbf{u}_{n}(\mathbf{x})\right), \mathbf{u}_{n}(\mathbf{x})\right)$. Then $\mathbf{h}_{n}\left(\mathbf{x}, \mathbf{u}_{n}(\mathbf{x})\right) \cos \beta_{n}(\mathbf{x})$ is uniformly integrable since

$$
\int_{E}\left|\mathbf{h}_{n}\left(\mathbf{x}, \mathbf{u}_{n}\right)\right|\left|\cos \beta_{n}\right| d \mathbf{x} \leq \frac{C_{11}}{M}+\int_{E} H_{M}(\mathbf{x}) d \mathbf{x},
$$

for any measurable subset $E$ of $\Omega$. Indeed, once we assume (2.22), given a $\varepsilon>0$, we can choose $\delta$ such that

$$
\int_{E} H_{M}(\mathbf{x}) d \mathbf{x}<\varepsilon \text { if } \operatorname{meas}(E)<\delta
$$


and taking $M$ such that $C_{11} / M<\varepsilon$, we get that

$$
\int_{E}\left|\mathbf{h}_{n}\left(\mathbf{x}, \mathbf{u}_{n}\right)\right|\left|\cos \beta_{n}\right| d \mathbf{x}<2 \varepsilon
$$

Then, by Vitali convergence theorem, we conclude that

$$
\mathbf{h}_{n}\left(., \mathbf{u}_{n}(.)\right) \cos \beta_{n}(.) \rightarrow \mathbf{h}(., \mathbf{u}(.)) \cos \beta(.) \text { in } \mathbf{L}^{1}(\Omega),
$$

which proves that

$$
\mathbf{h}_{n}\left(\mathbf{x}, \mathbf{u}_{n}\right) \rightarrow \mathbf{h}(\mathbf{x}, \mathbf{u}) \text { in } \mathbf{L}^{1}(\Omega) .
$$

Then $\mathbf{h}(\mathbf{x}, \mathbf{u}) \in \mathbf{L}^{1}(\Omega)$ and $\mathbf{u}$ is a weak solution to problem $\mathcal{P}\left(\Omega, \mathbf{u}_{*}, \mathbf{f}(\mathbf{x}, \mathbf{u})\right)$.

Uniqueness. Let $\mathbf{u}_{1}$ and $\mathbf{u}_{2}$ be two weak solutions. Then, according to Definition 2.1, $\mathbf{u}_{\mathbf{1}}-\mathbf{u}_{\mathbf{2}} \in \mathbf{H}_{0}^{1}(\Omega)$. Hence, $\mathbf{f}\left(\mathbf{x}, \mathbf{u}_{1}\right)-\mathbf{f}\left(\mathbf{x}, \mathbf{u}_{2}\right) \in \mathbf{H}^{-1}(\Omega)$. But, since $\left(\mathbf{f}\left(\mathbf{x}, \mathbf{u}_{1}\right)-\mathbf{f}\left(\mathbf{x}, \mathbf{u}_{2}\right)\right) \cdot\left(\mathbf{u}_{1}-\mathbf{u}_{2}\right) \leq 0$, we can use a vector version of a result due to Brezis and Browder [11] assuring that, if $T \in \mathrm{L}^{1}(\Omega) \cap \mathrm{H}^{-1}(\Omega)$ and $u \in \mathrm{H}^{1}(\Omega)$ are such that $T(\mathbf{x}) u(\mathbf{x}) \geq 0$ a.e. in $\Omega$, then $T u \in \mathrm{L}^{1}(\Omega)$ and

$$
\langle T, u\rangle_{\mathrm{H}^{-1}(\Omega) \times \mathrm{H}^{1}(\Omega)}=\int_{\Omega} T(\mathbf{x}) u(\mathbf{x}) d \mathbf{x} .
$$

Thus,

$$
\nu \int_{\Omega}\left|\nabla\left(\mathbf{u}_{\mathbf{1}}-\mathbf{u}_{\mathbf{2}}\right)\right|^{2} d \mathbf{x}=\int_{\Omega}\left(\mathbf{f}\left(\mathbf{x}, \mathbf{u}_{1}\right)-\mathbf{f}\left(\mathbf{x}, \mathbf{u}_{2}\right)\right) \cdot\left(\mathbf{u}_{1}-\mathbf{u}_{2}\right) d \mathbf{x}
$$

which implies that

$$
\int_{\Omega}\left|\nabla\left(\mathbf{u}_{1}-\mathbf{u}_{2}\right)\right|^{2} d \mathbf{x}=0
$$

and from Poincaré inequality (2.17) we get the result.

Remark 2.1 The above existence theorem admits many different variations $(\sigma \geq 1$, case of $\sigma=0$, more general unbounded sets $\Omega$, etc.), but they are not considered here. Our presentation is strictly motivated by the results on the localization effect.

\section{On the localization effect}

We recall that the existence of a weak solution having a finite global energy

$$
E:=\int_{\Omega}\left(|\nabla \mathbf{u}|^{2}+\chi_{\mathbf{f}}|u|^{1+\sigma}\right) d \mathbf{x}
$$

has been established in the previous section. The main result of this section is the following, where we consider the constant strip $(0, \infty) \times(0, L)$. 
Theorem 3.1 Assume that $\mathbf{f}$ satisfies (1.8) and (1.9). Then,

(i) if $x_{\mathbf{f}}=\infty$ ( $x_{\mathbf{f}}$ is given in (1.9)), $\mathbf{u}$ is any weak solution of $\mathcal{P}\left(\Omega, \mathbf{u}_{*}, \mathbf{f}\right)$ with finite energy $E$, then $\mathbf{u}(x, y)=\mathbf{0}$ for $x>a^{\prime}$, where $a^{\prime}=C(E, L, \delta, \nu, \sigma)$ is a positive constant;

(ii) if $x_{\mathbf{f}}<\infty$, then there exists at least one weak solution $\mathbf{u}$ of $\mathcal{P}\left(\Omega, \mathbf{u}_{*}, \mathbf{f}\right)$, with a finite energy $E$, such that if $a^{\prime}<x_{\mathbf{f}}$, then $\mathbf{u}(x, y)=\mathbf{0}$ for $x>a^{\prime}$;

(iii) if, in addition, we assume $\mathbf{f}$ non-increasing, then conclusion (ii) holds for the unique solution of $\mathcal{P}\left(\Omega, \mathbf{u}_{*}, \mathbf{f}\right)$.

In order to prove the localization effect, it is useful to work with the associated stream function $\psi$. We recall that due to the incompressibility condition (1.5), there exists a function $\psi$ such that

$$
u=\psi_{y} \text { and } v=-\psi_{x} \text { in } \Omega
$$

(see, e.g., Feistauer [16], Theorem 2.5.1). In this way, by classical methods (see, e.g., Ladyzhenskaya [23], Section 2.3), we can reduce the study of problem $\mathcal{P}\left(\Omega, \mathbf{u}_{*}, \mathbf{f}\right)$, to the consideration of problem $\mathcal{P}_{\psi}$ referred in (1.10), where the pressure term does not appear anymore.

The notion of weak solution is adapted to the information we have on function $\mathbf{f}\left(\mathbf{x}, \psi_{y},-\psi_{x}\right)$.

Definition 3.1 A function $\psi$ is called a weak solution of problem $\mathcal{P}_{\psi}$, if:

(i) $\psi \in \mathrm{H}^{2}(\Omega), \mathbf{f}\left(\mathbf{x}, \psi_{y},-\psi_{x}\right) \in \mathbf{L}^{1}(\Omega)$;

(ii) $\psi(0, y)=\int_{0}^{y} u_{*}(s) d s, \frac{\partial \psi}{\partial n}(0, y)=v_{*}(y)$ for $y \in(0, L), \psi(x, 0)=\psi(x, L)=$ $\frac{\partial \psi}{\partial n}(x, 0)=\frac{\partial \psi}{\partial n}(x, L)=0$ for $x \in(0, \infty)$ and $\psi(0,0)=\psi(0, L)=0 ;$

(iii) $\psi,|\nabla \psi| \rightarrow 0$, when $x \rightarrow \infty$;

(iv) $\nu \int_{\Omega} \triangle \psi \triangle \phi d \mathbf{x}-\int_{\Omega}\left(f_{1}\left(\mathbf{x}, \psi_{y},-\psi_{x}\right) \phi_{y}-f_{2}\left(\mathbf{x}, \psi_{y},-\psi_{x}\right) \phi_{x}\right) d \mathbf{x}=0$, for all $\phi \in \mathrm{H}_{0}^{2}(\Omega) \cap \mathrm{W}^{1, \infty}(\Omega)$ with compact support.

Lemma 3.1 If $\mathbf{u}$ is a weak solution of problem $\mathcal{P}\left(\Omega, \mathbf{u}_{*}, \mathbf{f}\right)$ in the sense of Definition 2.1, then $\psi$, given by (3.40), is a weak solution of problem $\mathcal{P}_{\psi}$ in the sense of Definition 3.1.

Proof. The only difficulty takes places with verifying (iv). However, given $\phi \in \mathrm{H}_{0}^{2}(\Omega) \cap \mathrm{W}^{1, \infty}(\Omega)$, we construct $\varphi=\left(\varphi_{1}, \varphi_{2}\right)$ with $\operatorname{div} \varphi=0$ such that $\phi$ is the current function associated to $\varphi=\left(\varphi_{1}, \varphi_{2}\right)$, i.e., $\varphi_{1}=\phi_{y}$ and $\varphi_{2}=-\phi_{x}$. Then we get

$$
\nu \int_{\Omega}\left(\psi_{y x} \phi_{y x}+\psi_{y y} \phi_{y y}+\psi_{x x} \phi_{x x}+\psi_{x y} \phi_{x y}\right) d \mathbf{x}-\int_{\Omega}\left(f_{1} \phi_{y}-f_{2} \phi_{x}\right) d \mathbf{x}=0 .
$$

Integrating by parts the first and fourth terms in the first integral, where we use the density of $\mathrm{C}_{0}^{\infty}(\Omega)$ in $\mathrm{H}_{0}^{2}(\Omega)$, we obtain the desired result.

Remark 3.1 The existence and uniqueness of a weak solution $\psi$ to problem $\mathcal{P}_{\psi}$ can be proved by different techniques without invoking to the problem $\mathcal{P}\left(\Omega, \mathbf{u}_{*}, \mathbf{f}\right)$. For some results of this nature see, e.g., Bernis [8], Grisvard [20], Lions [24] and the references therein. 
To establish the localization effect, as stated in Theorem 3.1, we will apply the so-called energy methods for free boundary problems (see [5]) introduced by Antontsev [1], improved by Díaz and Véron [14, 15], and extended by several authors amongst whom is Bernis [6].

We shall use here the technique of integrating over a family of variable halfplanes, which requires zero boundary conditions. We observe that the only non-zero boundary condition in problem $\mathcal{P}\left(\Omega, \mathbf{u}_{*}, \mathbf{f}\right)$, or problem $\mathcal{P}_{\psi}$, is on the boundary $x=0$. Thus, following [6], we are lead to introduce a weighted function which will cancel the terms on this boundary. For $m \geq 2$, let

$$
\psi(\mathbf{x})(x-a)_{+}^{m}=\left\{\begin{array}{cl}
0 & \text { if } x \leq a, \\
\psi(\mathbf{x})(x-a)^{m} & \text { if } x>a,
\end{array}\right.
$$

where $a \geq 0$ is a variable parameter and $\psi$ is a weak solution of $\mathcal{P}_{\psi}$. This function is not, in general, an admissible test function, because $\Omega$ is unbounded. Following Bernis [7], Appendix II, we approximate $\rho(x)=(x-a)_{+}^{m}$ by a sequence $\rho_{k}(x)=k^{m} \xi((x-a) / k)$, with $\xi \in \mathrm{C}(\mathbb{R}) \cap \mathrm{C}^{2}(0, \infty)$ and

$$
\xi(x)=\left\{\begin{array}{cl}
0 & \text { if } x \leq 0 \\
x^{m} & \text { if } 0<x \leq \frac{1}{2} \\
1 & \text { if } x \geq 1
\end{array}\right.
$$

Due to the fact that $m \geq 2$, it is possible to show that $\rho_{k} \in \mathrm{W}^{2, \infty}(\Omega)$ and thus $\psi(\mathbf{x}) \rho_{k}(x)$ is already a test function. Moreover

$$
0 \leq \mathrm{D}^{i} \rho_{1}(x) \leq \mathrm{D}^{i} \rho_{2}(x) \leq \ldots \leq \mathrm{D}^{i} \rho_{k}(x) \leq \mathrm{D}^{i} \rho_{k+1}(x) \leq \ldots
$$

and

$$
\mathrm{D}^{i} \rho_{k}(x) \rightarrow m(m-1) \ldots[m-(i-1)](x-a)_{+}^{m-i}, \quad \text { as } k \rightarrow \infty,
$$

for all $x>0, m \geq 2,0 \leq i \leq m$ and $i \leq 2$. Thus, we can prove the following result.

Lemma 3.2 Let $\psi$ be a weak solution of $\mathcal{P}_{\psi}$ with $E$ finite. Assume that $\mathbf{f}$ satisfies (1.8) and (1.9) with $x_{\mathbf{f}}=\infty$. Then, for every $a>x_{g}$ and every positive integer $m \geq 2$,

$$
\begin{gathered}
\min (\nu, \delta) \int_{\Omega}\left(\left|\mathrm{D}^{2} \psi\right|^{2}+\left|\psi_{y}\right|^{1+\sigma}\right)(x-a)_{+}^{m} d \mathbf{x} \leq \\
2 m \nu \int_{\Omega}|\triangle \psi|\left|\psi_{x}\right|(x-a)_{+}^{m-1} d \mathbf{x}+2 m \nu \int_{\Omega}\left|\psi_{y}\right|\left|\psi_{x y}\right|(x-a)_{+}^{m-1} d \mathbf{x} \\
+m(m-1) \nu \int_{\Omega}|\triangle \psi||\psi|(x-a)_{+}^{m-2} d \mathbf{x},
\end{gathered}
$$

where $\left|\mathrm{D}^{2} \psi\right|^{2}=\psi_{x x}^{2}+2 \psi_{x y}^{2}+\psi_{y y}^{2}$. 
Proof. Taking $\psi(\mathbf{x}) \rho_{k}(x)$ as a test function in Definition 3.1, applying the Leibnitz formula and using assumptions (1.8) and (1.9), we obtain

$$
\begin{aligned}
& \nu \int_{\Omega}(\triangle \psi)^{2} \rho_{k} d \mathbf{x}+\delta \int_{\Omega}\left|\psi_{y}\right|^{1+\sigma} \rho_{k} d \mathbf{x} \leq \\
& -2 \nu \int_{\Omega} \triangle \psi \psi_{x} \rho_{k}^{\prime} d \mathbf{x}-\nu \int_{\Omega} \triangle \psi \psi \rho_{k}^{\prime \prime} d \mathbf{x} .
\end{aligned}
$$

The study of the first term on the left-hand side requires an integration by parts leading to

$$
\int_{\Omega} \psi_{x x} \psi_{y y} \rho_{k} d \mathbf{x}=\int_{\Omega} \psi_{x y}^{2} \rho_{k} d \mathbf{x}+\int_{\Omega} \psi_{y} \psi_{x y} \rho_{k}^{\prime} d \mathbf{x},
$$

where we used a regularization procedure on $\psi$. Then from (3.44), it comes

$$
\begin{gathered}
\nu \int_{\Omega}\left|\mathrm{D}^{2} \psi\right|^{2} \rho_{k} d \mathbf{x}+\delta \int_{\Omega}\left|\psi_{y}\right|^{1+\sigma} \rho_{k} d \mathbf{x} \leq \\
-2 \nu \int_{\Omega} \triangle \psi \psi_{x} \rho_{k}^{\prime} d \mathbf{x}-2 \nu \int_{\Omega} \psi_{y} \psi_{x y} \rho_{k}^{\prime} d \mathbf{x}-\nu \int_{\Omega} \triangle \psi \psi \rho_{k}^{\prime \prime} d \mathbf{x} .
\end{gathered}
$$

We take the minimum on the left-hand side and apply modules. Finally, (3.43) follows from (3.41), (3.42) and the theorem on monotone convergence.

In the first term of left-hand side of the inequality (3.43), it arises the energy type term which depends on $a$

$$
E_{m}(a)=\int_{\Omega}\left(\left|\mathrm{D}^{2} \psi\right|^{2}+\left|\psi_{y}\right|^{1+\sigma}\right)(x-a)_{+}^{m} d \mathbf{x}
$$

We observe that $E_{0}(0)=E$ (recall that $\left.x_{\mathbf{f}}=\infty\right)$.

The mentioned technique, as introduced in [6], has, as main goal, to get a differential inequality for $E_{m}(a)$ leading to the vanishing of $E_{m}(a)$ (and then of $\psi$ ) for $a$ large enough. Notice that a simple differentiation leads to the relations

$$
\frac{d E_{m}(a)}{d a}=-m E_{m-1}(a) \text { and } \frac{d^{2} E_{m}(a)}{d a^{2}}=m(m-1) E_{m-2}(a)
$$

The crucial part of the technique consists in to use the non-linear structure of the equation in order to get some differential inequality. To this end, a fundamental role will be played by two inequalities. The first, is a weighted Gagliardo-Nirenberg inequality derived in [6], Appendix I, from Nirenberg [25], Lecture II.

Lemma 3.3 If $j, k, l$ are integers with $0 \leq j<k, k \geq 1$ and $l \geq 0$ and $1 \leq p<\infty$ and if $1 \leq r \leq p$, then

$$
\begin{gathered}
\int_{\Omega}\left|D^{j} u\right|^{p}(x-a)^{l} d \mathbf{x} \leq \\
C_{12}\left(\int_{\Omega}\left|D^{k} u\right|^{p}(x-a)^{l} d \mathbf{x}\right)^{\theta}\left(\int_{\Omega}|u|^{r}(x-a)^{l} d \mathbf{x}\right)^{p \frac{1-\theta}{r}},
\end{gathered}
$$


once that the integrals of the right-hand side exist, where $\theta$ is given by

$$
\frac{1}{p}=\frac{j}{2+l}+\theta\left(\frac{1}{p}-\frac{k}{2+l}\right)+(1-\theta) \frac{1}{r}
$$

and $C_{12}=C_{12}(j, k, l, p, r)$.

Here, $\mathrm{D}^{k} u$ denotes the vector of all derivatives of order $k$, with $k$ integer non-negative and

$$
\left|\mathrm{D}^{k} u\right|^{2}=\sum_{|\alpha|=k}\left|\mathrm{D}^{\alpha} u\right|^{2} \text {, where } \mathrm{D}^{\alpha} u=\frac{\partial^{|\alpha|} u}{\partial^{\alpha_{1}} x \partial^{\alpha_{2}} y} \text { and }|\alpha|=\alpha_{1}+\alpha_{2} \text {. }
$$

The second, is a Hardy type inequality derived in [6], Appendix I , from Hardy et al [21], Theorem 330.

Lemma 3.4 If $1<p<\infty, l \geq 0$ and $u$ is bounded in a neighborhood of $x=0$, then

$$
\int_{\Omega}|u|^{p}(x-a)^{l} d \mathbf{x} \leq\left(\frac{p}{l+1}\right)^{p} \int_{\Omega}\left|u_{x}\right|^{p}(x-a)^{l+p} d \mathbf{x},
$$

once that the integrals of both sides exist.

After the differential inequality has been obtained, the following auxiliary result, a direct consequence of [7], Appendix III, leads us to the conclusion.

Lemma 3.5 Assume that the fractional differential inequality

$$
E_{m}(a) \leq C\left(E_{m-p}(a)\right)^{\mu}
$$

holds for all $a \geq x_{g}>0$, where $0<p \leq m<w, C$ is a positive constant and $\mu>1$. Assume $E_{m-p}(a)$ is finite for any $a \geq x_{g}$. Then, the support of $E_{0}(a)$ is a bounded interval $\left[0, a^{*}\right]$ with $a^{*} \leq a^{\prime}$ and where

$$
a^{\prime}=(w-m+1) C^{\frac{1}{(\mu-1)(w-m)}} E^{\frac{1}{w-m}} \quad \text { and } \quad w=\frac{\mu p}{\mu-1} .
$$

So, we arrive at the more difficult part of the proof of Theorem 3.1.

Lemma 3.6 Let $\psi$ be a weak solution of $P_{\psi}$ and assume $\mathbf{f}$ satisfies (1.8) and (1.9) with $x_{\mathbf{f}}=\infty$. Then, the following differential inequalities hold for $a \geq x_{g}$ :

$$
E_{m}(a) \leq C_{13}\left(E_{m-2}(a)\right)^{\mu}, \text { for every integer } m>3
$$

and

$$
E_{2}(a) \leq C E_{0}(a)+C_{14}\left(E_{0}(a)\right)^{\mu}, \quad \text { if } m=2 ;
$$

where $C_{13}=C_{13}(L, m, \delta, \nu, \sigma), C_{14}=C_{14}(L, \delta, \nu, \sigma)$ and $\mu=\mu(m, \sigma)$ are positive constants, with $C$ an absolute constant, $\mu>1$ and $x_{g}$ given in (1.9). 
Proof. We rewrite (3.43) as

$$
\min (\nu, \delta) E_{m}(a) \leq 2 m \nu I_{1}+2 m \nu I_{2}+m(m-1) \nu I_{3} .
$$

Now, let us estimate $I_{1}, I_{2}$ and $I_{3}$ in terms of $E_{m}(a)$ and $E_{m-2}(a)$.

At this point, we have to distinguish two different cases.

Case $m>3$. Applying Cauchy's inequality with $\varepsilon \in(0,1)$ to each one of these terms and then adding up the connected terms, (3.50) comes

$$
\min (\nu, \delta) E_{m}(a) \leq m(m+1) \nu \varepsilon E_{m}(a)+\frac{m \nu}{\varepsilon} I_{22}+\frac{m(m-1) \nu}{2 \varepsilon} I_{32},
$$

where

$$
I_{22}:=\int_{\Omega}|\nabla \psi|^{2}(x-a)_{+}^{m-2} d \mathbf{x} \text { and } I_{32}:=\int_{\Omega} \psi^{2}(x-a)_{+}^{m-4} d \mathbf{x} .
$$

Applying the weighted Gagliardo-Nirenberg inequality (3.45) to $I_{22}$ for the function $\psi$, with $j=1, k=2, l=m-2, p=2$ and $r=1+\sigma$, we get

$$
I_{22} \leq C_{15}\left(\int_{\Omega}\left|\mathrm{D}^{2} \psi\right|^{2}(x-a)_{+}^{m-2} d \mathbf{x}\right)^{\theta}\left(\int_{\Omega}|\psi|^{1+\sigma}(x-a)_{+}^{m-2} d \mathbf{x}\right)^{2 \frac{1-\theta}{1+\sigma}},
$$

where

$$
\theta=\frac{2(1+\sigma)+(1-\sigma) m}{4(1+\sigma)+(1-\sigma) m}
$$

and $C_{15}=C_{15}(m, \sigma)$. Notice that $0<\theta<1$, because $0<\sigma<1$. Applying the Poincare inequality (2.17), with $p=1+\sigma$ to the term $\int_{0}^{L}|\psi|^{1+\sigma} d y$ and then the inequality $A^{\alpha} B^{\beta} \leq(A+B)^{\alpha+\beta}$, where $A, B \geq 0$,

$$
I_{22} \leq C_{15}\left(\frac{L}{\pi}\right)^{2(1-\theta)}\left(E_{m-2}(a)\right)^{\mu},
$$

where

$$
\mu=1+\frac{2(1-\sigma)}{4(1+\sigma)+(1-\sigma) m}>1,
$$

because $0<\sigma<1$. Now, applying the Hardy type inequality (3.46) to $I_{32}$ for the function $\psi$, with $l=m-4$ and $p=2$

$$
I_{32} \leq\left(\frac{2}{m-3}\right)^{2} \int_{\Omega}\left|\psi_{x}\right|^{2}(x-a)_{+}^{m-2} d \mathbf{x} .
$$

Because $\left|\psi_{x}\right|^{2} \leq|\nabla \psi|^{2}, I_{32} \leq 4 /(m-3)^{2} I_{22}$, then from (3.54)

$$
I_{32} \leq \frac{4 C_{15}}{(m-3)^{2}}\left(\frac{L}{\pi}\right)^{2(1-\theta)}\left(E_{m-2}(a)\right)^{\mu},
$$


where $\theta$ and $\mu$ are given by (3.53) and (3.55), respectively. Then, from (3.54) and (3.56), (3.51) comes

$$
\min (\nu, \delta) E_{m}(a) \leq \varepsilon C_{16} E_{m}(a)+\frac{1}{\varepsilon} C_{17}\left(E_{m-2}(a)\right)^{\mu},
$$

where $C_{16}=C_{16}(m, \nu), C_{17}=C_{17}(L, m, \nu, \sigma)$ and $m>3$. Choosing $\varepsilon=$ $\min (\nu, \delta) /\left(2 C_{16}\right)$, we achieve to the fractional inequality (3.48), which is valid for every $m>3$ and where

$$
C_{13}=4 C_{15} \frac{m^{2}(m+1)\left(m^{2}-4 m+7\right)}{(m-3)^{2}} \frac{\nu^{2}}{\min ^{2}(\nu, \delta)}\left(\frac{L}{\pi}\right)^{2(1-\theta)}
$$

and $C_{15}$, given immediately after to (3.53), is the constant resulting from Gagliardo-Nirenberg inequality (3.45).

Case $m=2$. In this case, we have only to worry about with the term $I_{3}$, because for the other terms the above estimates remain valid. For $m=2$,

$$
I_{3}=\int_{\Omega_{a}} \triangle \psi \psi d \mathbf{x}
$$

where $\Omega_{a}=(a, \infty) \times(0, L)$. Applying Cauchy's inequality with $\varepsilon \in(0,1)$ to $I_{3}$ and proceeding in the same manner for the other terms as in the preceding case, but taking $m=2$, we obtain the analogous inequality from (3.51)

$$
\min (\nu, \delta) E_{2}(a) \leq 4 \nu \varepsilon E_{2}(a)+2 \nu \varepsilon E_{0}(a)+\frac{2 \nu}{\varepsilon} I_{22(m=2)}+\frac{\nu}{\varepsilon} I_{32(m=2)},
$$

where

$$
I_{22(m=2)}:=\int_{\Omega_{a}}|\nabla \psi|^{2} d \mathbf{x} \text { and } I_{32(m=2)}:=\int_{\Omega_{a}} \psi^{2} d \mathbf{x} .
$$

Taking $m=2$ in (3.54),

$$
I_{22(m=2)} \leq C_{18}\left(\frac{L}{\pi}\right)^{2(1-\theta)}\left(E_{0}(a)\right)^{\mu},
$$

where, from (3.53) and (3.55), respectively,

$$
\theta=\frac{2}{3+\sigma} \text { and } \mu=\frac{4}{3+\sigma}
$$

and $C_{18}=C_{15}$ with $m=2$. Applying Poincaré inequality (2.17) to the term $\int_{0}^{L}|\psi|^{2} d y$ of $I_{32(m=2)}, I_{32(m=2)} \leq(L / \pi)^{2} I_{22(m=2)}$ and from (3.60),

$$
I_{32(m=2)} \leq C_{18}\left(\frac{L}{\pi}\right)^{2(1-\theta)+2}\left(E_{0}(a)\right)^{\mu} .
$$

Then, (3.60) and (3.62), (3.58) yield that

$$
\min (\nu, \delta) E_{2}(a) \leq \varepsilon C_{19}\left(E_{2}(a)+E_{0}(a)\right)+\frac{1}{\varepsilon} C_{20}\left(E_{0}(a)\right)^{\mu},
$$


where $C_{19}=C_{19}(\nu), C_{20}=C_{20}(L, \nu, \sigma)$. Now, if we choose $\varepsilon=\min (\nu$, $\delta) /\left(2 C_{19}\right)$ in $(3.63)$, we come to the differential inequality (3.49), where

$$
C=\frac{1}{2}, \quad C_{14}=16 C_{18} \frac{\nu^{2}}{\min ^{2}(\nu, \delta)}\left[2+\left(\frac{L}{\pi}\right)^{2}\right]\left(\frac{L}{\pi}\right)^{2(1-\theta)},
$$

with $\theta$ and $\mu$ given by (3.61) and $C_{18}$ immediately after

Proof of Theorem 3.1. We start with the case $x_{\mathbf{f}}=\infty$. Taking $m=4$ in Lemma 3.6, we have the fractional differential inequality

$$
E_{4}(a) \leq C_{21}\left(E_{2}(a)\right)^{\mu},
$$

where from (3.53), (3.55) and (3.57), respectively,

$$
\theta=\frac{3-\sigma}{4}, \mu=\frac{5-\sigma}{4} \text { and } C_{21}=2240 C_{22} \frac{\nu^{2}}{\min ^{2}(\nu, \delta)}\left(\frac{L}{\pi}\right)^{\frac{1+\sigma}{2}}
$$

and $C_{22}=C_{15}$ with $m=4$. One can easily see that, due to $0<\sigma<1$, $1 / 2<\theta<3 / 4$ and $1<\mu<5 / 4$. Using Lemma 3.6 with $m=2$ and because of the finiteness of $E$, we can easily see that $E_{2}(a)$ is finite. Then from Lemma 3.5, with $m=4, p=2$ and $w=8 /(1-\sigma)+2>4=m$, the support of $E_{0}(a)$ is a bounded interval $\left[0, a^{*}\right]$ with $a^{*} \leq a^{\prime}$ and where, from (3.47) and (3.64),

$$
a^{\prime}=\frac{7+\sigma}{1-\sigma} C_{21}^{\frac{2}{3+\sigma}} E^{\frac{1-\sigma}{2(3+\sigma)}} .
$$

Then

$$
E_{0}(a)=\int_{\Omega_{a}}\left(|\nabla \mathbf{u}|^{2}+|u|^{1+\sigma}\right) d \mathbf{x}=0
$$

for $a>a^{\prime}$, which implies $u=0$ and $v$ is constant almost everywhere for $x>a^{\prime}$. Finally, from (1.2), $v=0$ in the same domain.

Assume now that $x_{\mathbf{f}}<\infty$. Then we construct a weak solution in the following way

$$
\mathbf{u}(\mathbf{x})=\left\{\begin{array}{cl}
\widetilde{\mathbf{u}}(\mathbf{x}) & \text { if } x \leq a^{\prime} \\
\mathbf{0} & \text { if } x>a^{\prime}
\end{array}\right.
$$

with $\widetilde{\mathbf{u}}(\mathbf{x})$ weak solution of $\mathcal{P}\left(\Omega, \mathbf{u}_{*}, \mathbf{f}\right)$ with $x_{\mathbf{f}}=\infty$. By the proof of the above case and the assumption $a^{\prime}<x_{\mathbf{f}}$, we get that $\mathbf{u}(\mathbf{x})$ is a weak solution of the original problem $\mathcal{P}\left(\Omega, \mathbf{u}_{*}, \mathbf{f}\right)$

Remark 3.2 Obviously, we obtain an analogous localization effect if we replace the role of variables $x$ and $y$ for the study of unbounded sets of the form $\Omega=$ $(0, L) \times(0, \infty)$, and if we modify correspondingly the condition(1.8).

Remark 3.3 In the case of $\sigma=1$, the above arguments lead to the inequality

$$
E_{m}(a) \leq C E_{m-2}(a), \text { for } a \geq x_{g},
$$

and then an exponential decay is derived (this type of decay is optimal). The exponential decay estimate is derived using differential inequality techniques analogous to those developed by Knowles [22] and Toupin [28] in their energy approach to the investigation of the Saint-Venant's Principle in classical elasticity theory. 
Remark 3.4 The localization effect can be extended to the limit case $\sigma=0$ following the approach presented in Diaz [12].

Remark 3.5 The main result of this section can be applied to the family of stationary problems obtained by implicit discretization of the parabolic problem

$$
\begin{cases}\frac{\mathbf{u}_{n}-\mathbf{u}_{n-1}}{\tau}-\nu \triangle \mathbf{u}_{n}=\mathbf{f}\left(\mathbf{x}, \mathbf{u}_{n}\right)-\nabla p_{n} & \text { in } \Omega \\ \operatorname{div} \mathbf{u}_{n}=0 & \text { in } \Omega\end{cases}
$$

A direct proof of the localization effect for the non-discretized parabolic problem will be given by the authors in [4].

\section{The non-constant semi-infinite strip}

In this section we prove that the localization effect holds for domains of the type $\Omega=(0, \infty) \times\left(L_{1}(x), L_{2}(x)\right)$, where the functions $L_{1}$ and $L_{2}$ are smooth enough and $L_{1}(x) \neq L_{2}(x)$ for all $x \geq 0$. In this case, equations (1.4) and (1.5) are appended with the boundary conditions (2.13) and (2.14) and the compatibility conditions are, now, (2.15) and (2.16). The condition at infinity is the same, (1.3). The assumptions on $\mathbf{f}$ are, mutatis mutandis, the same, (1.8) and (1.9). The main difficulty in this case is the applicability of the weighted GagliardoNirenberg inequality (3.45) and the Hardy type inequality (3.46). But, as it is known, these inequalities still hold for any domain which can be mapped, by a sufficiently smooth mapping, in a one-to-one way onto a product-like domain. So, if we consider $\Omega$ such regular, we can prove the same localization effects for any weak solution of this problem. The correspondent result of Lemma 3.6 is the following one.

Lemma 4.1 Let $\psi$ be a weak solution of $\mathcal{P}_{\psi}$ and assume $\mathbf{f}$ satisfies the adapted conditions (1.8) and (1.9) for this case. Assume also (2.11), (2.12) and additionally

$$
\begin{aligned}
\left|L_{1}^{\prime}(x)\right|,\left|L_{2}^{\prime}(x)\right| & \leq L^{\prime}, \\
\left|L_{1}^{\prime \prime}(x)\right|,\left|L_{2}^{\prime \prime}(x)\right| & \leq L^{\prime \prime},
\end{aligned}
$$

for any $x \geq 0$, where $L^{\prime}$ and $L^{\prime \prime}$ are positive constants. Then, the following differential inequalities hold for $a \geq x_{g}$ :

$$
E_{m}(a) \leq C_{23}\left(E_{m-2}(a)\right)^{\mu}, \text { for every integer } m>3
$$

and

$$
E_{2}(a) \leq C E_{0}(a)+C_{24}\left(E_{0}(a)\right)^{\mu}, \text { if } m=2 ;
$$

where $C_{23}=C_{23}\left(L, L^{\prime}, L^{\prime \prime}, m, \delta, \nu, \sigma\right), C_{24}=C\left(L, L^{\prime}, L^{\prime \prime}, \delta, \nu, \sigma\right)$ and $\mu=\mu(m$, $\sigma)$ are positive constants, with $C$ an absolute constant, $\mu>1$ and $x_{g}$ given in (1.9). 
Proof. Case $m>3$. Proceeding as we did in the proof of Lemma 3.6 and using the assumptions (1.8) and (1.9), we obtain the analogous of (3.51)

$$
\min (\nu, \delta) E_{m}(a) \leq m(m+1) \nu \varepsilon E_{m}(a)+\frac{m \nu}{\varepsilon} I_{22}+\frac{m(m-1) \nu}{2 \varepsilon} I_{32},
$$

where $I_{22}$ and $I_{32}$ are given in (3.52) with $\Omega=(0, \infty) \times\left(L_{1}(x), L_{2}(x)\right)$. Let us make the following change of variables $\mathbf{x}=\boldsymbol{\xi}, \boldsymbol{\xi}=(\xi, \eta)$, on $I_{22}$ and on $I_{32}$,

$$
x=\xi \text { and } y=\frac{L_{2}(\xi)-L_{1}(\xi)}{L} \eta+L_{1}(\xi),
$$

where $L$ is a positive constant and $\boldsymbol{\xi} \in \Omega(\boldsymbol{\xi})=(0, \infty) \times(0, L)$. Notice that (2.11) and (2.12) makes this possible and we have

$$
\psi_{x}=\psi_{\xi}-y_{\xi} y_{\eta}^{-1} \psi_{\eta} \text { and } \psi_{y}=y_{\eta}^{-1} \psi_{\eta}
$$

Using (4.71) and assumptions (2.12), (4.65) and (4.66) on $I_{22}$ and on $I_{32}$ and then using (3.54) and (3.56), we obtain from (4.69),

$$
\min (\nu, \delta) E_{m}(a) \leq \varepsilon C_{25} E_{m}(a)+\frac{1}{\varepsilon} C_{26}\left(\mathcal{E}_{m-2}(a)\right)^{\mu},
$$

where $C_{25}=C_{25}(m, \nu), C_{26}=C_{26}\left(L, L^{\prime}, m, \nu, \sigma\right), m>3$ and

$$
\mathcal{E}_{m-2}(a)=\int_{\Omega(\boldsymbol{\xi})}\left(\left|\mathrm{D}^{2} \psi(\xi, \eta)\right|^{2}+\left|\psi_{\eta}(\xi, \eta)\right|^{1+\sigma}\right)(\xi-a)_{+}^{m-2} d \boldsymbol{\xi} .
$$

Now, we come back to the original variables $(x, y)$ making the inverse change of variables of (4.70) in $\mathcal{E}_{m-2}(a)$

$$
\xi=x \text { and } \eta=L \frac{y-L_{1}(x)}{L_{2}(x)-L_{1}(x)} .
$$

After we made use of Cauchy's inequality, assumptions (2.12), (4.65) and (4.66) and Poincaré inequality (2.17), (4.72) comes

$$
\min (\nu, \delta) E_{m}(a) \leq \varepsilon C_{25} E_{m}(a)+\frac{1}{\varepsilon} C_{27}\left(E_{m-2}(a)\right)^{\mu},
$$

where, now, $C_{27}=C_{27}\left(L, L^{\prime}, L^{\prime \prime}, m, \nu, \sigma\right)$ and $m>3$. Choosing $\varepsilon=\min (\nu, \delta) /$ $\left(2 C_{25}\right)$, we come to the fractional differential inequality (4.67), where

$$
C_{23}=4 \gamma C_{15} \frac{m^{2}(m+1)\left(m^{2}-4 m+7\right)}{(m-3)^{2}} \frac{\nu^{2}}{\min ^{2}(\nu, \delta)}\left(\frac{L}{\pi}\right)^{2(1-\theta)}
$$

with $\theta$ given by (3.53), $\mu$ by (3.55), $C_{15}$ immediately after to (3.53) and

$$
\gamma=\gamma\left(L, L^{\prime}, L^{\prime \prime}, \sigma\right)
$$

results from the changes of variables (4.70) and (4.73). 
Case $m=2$. Here, the analogous of $(3.58)$ is

$$
\min (\nu, \delta) E_{2}(a) \leq 4 \nu \varepsilon E_{2}(a)+2 \nu \varepsilon E_{0}(a)+\frac{2 \nu}{\varepsilon} I_{22(m=2)}+\frac{\nu}{\varepsilon} I_{32(m=2)},
$$

where $I_{22(m=2)}$ and $I_{32(m=2)}$ are given in (3.59) with $\Omega_{a}=(a, \infty) \times\left(L_{1}(x)\right.$, $\left.L_{2}(x)\right)$. For $I_{22(m=2)}$ we can obtain an estimate in the same manner as for the previous case. For $I_{32(m=2)}$, we use Poincaré's inequality (2.17) in order to obtain $I_{22(m=2)}$ and then we can use the already known estimate. Then, we come back to the original variables, by the change of variables (4.73). Thus, we obtain from (4.75),

$$
\min (\nu, \delta) E_{2}(a) \leq \varepsilon C_{28}\left(E_{2}(a)+E_{0}(a)\right)+\frac{1}{\varepsilon} C_{29}\left(E_{0}(a)\right)^{\mu},
$$

where $C_{28}=C_{28}(\nu), C_{29}=C_{29}\left(L, L^{\prime}, L^{\prime \prime}, \nu, \sigma\right)$. Now, choosing $\varepsilon=\min (\nu, \delta) /$ $\left(2 C_{28}\right)$,we achieve to $(4.68)$, where

$$
C=\frac{1}{2}, \quad C_{24}=16 \gamma C_{18}\left[2+\left(\frac{L}{\pi}\right)^{2}\right] \frac{\nu^{2}}{\min ^{2}(\nu, \delta)}\left(\frac{L}{\pi}\right)^{2(1-\theta)},
$$

where $\theta, C_{18}$ and $\mu$ are given by (3.61) and $\gamma$ is given by (4.74).

Using exactly the same justifications that we did in the proof of Theorem 3.1, we can prove the following result.

Theorem 4.1 Assume f satisfies the adapted conditions (1.8) and (1.9) for this case. Assume also (2.11)-(2.12) and (4.65)-(4.66) are satisfied. Then:

(i) if $x_{\mathbf{f}}=\infty\left(x_{\mathbf{f}}\right.$ is given in (1.9)), $\mathbf{u}$ is any weak solution of $\mathcal{P}\left(\Omega, u_{*}, \mathbf{f}\right)$ with finite energy $E$, then $\mathbf{u}(x, y)=\mathbf{0}$ for $x>a_{\mathrm{nc}}^{\prime}$, where

$$
a_{\mathrm{nc}}^{\prime}=\frac{7+\sigma}{1-\sigma} C_{30}^{\frac{2}{3+\sigma}} E^{\frac{1-\sigma}{2(3+\sigma)}}, \quad C_{30}=C_{31} \frac{\nu^{2}}{\min ^{2}(\nu, \delta)}\left(\frac{L}{\pi}\right)^{\frac{1+\sigma}{2}}
$$

with $C_{31}=C_{31}\left(L, L^{\prime}, L^{\prime \prime}, \sigma\right)$ a positive constant;

(ii) if $x_{\mathbf{f}}<\infty$, then there exists at least one weak solution $\mathbf{u}$ of $\mathcal{P}\left(\Omega, u_{*}, \mathbf{f}\right)$, with a finite energy $E$, such that if $a_{\mathrm{nc}}^{\prime}<x_{\mathbf{f}}$, then $\mathbf{u}(x, y) \equiv \mathbf{0}$ for $x>a_{\mathrm{nc}}^{\prime}$;

(iii) if, in addition, we assume $\mathbf{f}$ non-increasing then conclusion (ii) holds for the unique solution of $\mathcal{P}\left(\Omega, u_{*}, \mathbf{f}\right)$.

\section{References}

[1] S.N. Antontsev. On the localization of solutions of non-linear degenerate elliptic and parabolic equations (Russian). Dokl. Akad. Nauk SSSR, 260 no.6 (1981), 1289-1293. English translation in Dokl. Math., 24 no.2 (1981), $420-424$.

[2] S.N. Antontsev, J.I. Díaz, H.B. de Oliveira. On the confinement of a viscous fluid by means of a feedback external field. To appear in C. R. Mecanique Acad. Sci. Paris. 
[3] S.N. Antontsev, J.I. Díaz, H.B. de Oliveira. Stopping a viscous fluid by a feedback dissipative external field: II. The stationary Navier-Stokes problem. In preparation.

[4] S.N. Antontsev, J.I. Díaz, H.B. de Oliveira. Stopping a viscous fluid by a feedback dissipative external field: III. The evolution Navier-Stokes problem. In preparation.

[5] S.N. Antontsev, J.I. Díaz, S.I. Shmarev. Energy Methods for Free Boundary Problems: Applications to non-linear PDEs and fluid mechanics. Birkhäuser, Boston, 2002.

[6] F. Bernis. Compactness of the support for some non-linear elliptic problems of arbitrary order in dimension n. Commun. Partial Diff. Equations, 9 no.3 (1984), 271-312.

[7] F. Bernis. Qualitative properties for some non-linear higher order degenerate parabolic equations, Houston J. Math., 14 (1988), 319-352.

[8] F. Bernis. Elliptic and Parabolic Semilinear Problems without Conditions at Infinity, Arch. Ration. Mech. Anal., 106 (1989), 217-241.

[9] H. Berstycki, H. Brezis. On a free boundary problem arising in plasma physics. Nonlinear Anal., 4 (1980), 415-436.

[10] H. Brezis, F.E. Browder. Strongly non-linear elliptic boundary value problems. Ann. Scuola Norm. Sup. Pisa Cl. Sci. (IV), 5 (1978), 587-603.

[11] H. Brezis, F.E. Browder. Some properties of higher order Sobolev spaces. J. Math. Pures Appl. 61 (1982), 245-259.

[12] J.I. Díaz. On the formation of the free boundary for the obstacle and Stefan problems via an energy method. In the CD-Rom Actas XVII CEDYA / VII CMA. L. Ferragut y A. Santos eds., S.P. Universidad Salamanca, 2001.

[13] J.I. Díaz, J.M. Rakotoson. On a nonlocal stationary free boundary problem arising in the confinement of a plasma in a Stellarator geometry. Arch. Ration. Mech. Anal., 134 (1996), 53-95

[14] J.I. Díaz, L. Véron. Compacité du support des solutions d'équations quasi linéaires elliptiques ou paraboliques. C. R. Acad. Sci. Paris Ser. I Math., 297 no.3 (1983), 149-152.

[15] J.I. Díaz, L. Véron. Local vanishing properties of solutions of elliptic and parabolic quasilinear equations. Trans. Am. Math. Soc., 290 no.2 (1985), 787-814.

[16] M. Feistauer. Mathematical Methods in Fluid Dynamics. Pitman Longman, Harlow, 1993. 
[17] J.P. Freidberg. Ideal magnetohydrodinamic theory of magnetic fusion systems. Rev. Mod. Phys., 54, no. 3, July 1982.

[18] G.P. Galdi. An Introduction to the Mathematical Theory of the NavierStokes Equations: Linearised Steady Problems. Springer-Verlag, New York, 1994.

[19] D. Gilbarg, N.S. Trudinger. Elliptic Partial Differential Equations of Second Order. Springer-Verlag, Berlin Heidelberg, 1998.

[20] P. Grisvard. Elliptic Problems in Nonsmooth Domains. Pitman, Boston, 1985.

[21] G. Hardy, J.E. Littlewood, G. Pólya. Inequalities. Cambridge University Press, Cambridge, 1997 (reprint of the 1952 edition).

[22] J.K. Knowles. On Saint-Venant's Principle in the Two-Dimensional Linear Theory of Elasticity. Arch. Ration. Mech. Anal., 21 (1966), 1-22.

[23] O.A. Ladyzhenskaya. The mathematical theory of viscous incompressible flow. Gordon and Breach, New York, 1969 (2nd English edition).

[24] J.L. Lions. Quelques Méthodes de resolution des problémes aux limites non lineaires. Dunod, Paris, 1969.

[25] L. Nirenberg. On elliptical partial differential equations. Ann. Scuola Norm. Sup. Pisa Cl. Sci. (IV) 13 (1959), 115-162.

[26] J. Simon. Asymptotic behaviour of a plasma induced by an electric current. Nonlinear Anal. (1985), 149-169.

[27] R. Temam. Remarks on a free boundary problem arising in Plasma Physics. Comm. Partial Diff. Equations, 2 (1977), 563-585.

[28] R.A. Toupin. Saint-Venant's Principle. Arch. Ration. Mech. Anal., 18 (1965), 83-96.

[29] I.I. Vrabie. Compactness methods for non-linear evolutions. Pitman Longman, London, 1987. 